Andreas Gardt

\title{
Text und Bedeutung
}

(vorgetragen in der Plenarsitzung am 17. April 2009)

Mein akademischer Werdegang ist in der Studienphase mit den Universitäten Mainz, Heidelberg, East Anglia und Cambridge verbunden, in der Phase der Berufstätigkeit mit den Universitäten Heidelberg, Reading, Osnabrück, Freiburg, schließlich Kassel. Während des Studiums der Germanistik und Anglistik in Heidelberg und eines MasterStudiums der Comparative Litera-

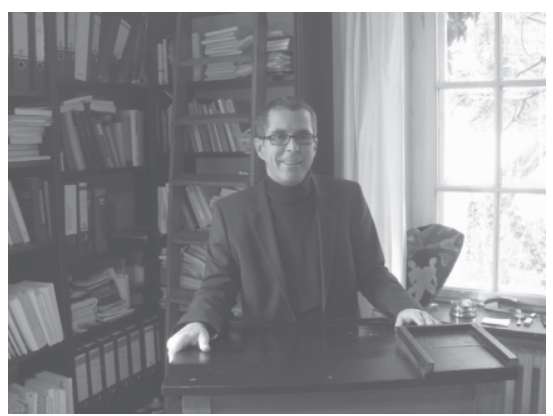

Andreas Gardt, Professor für Sprachwissenschaften an der Universität Kassel, O. Mitglied der Göttinger Akademie seit 2009 ture an der University of East Anglia kam ich erstmals mit Themen aus dem Übergangsbereich von Sprach- und Literaturwissenschaft in Kontakt. So fand die Promotion in der anglistischen Literaturwissenschaft statt (1987), die Habilitation in der germanistischen Sprachwissenschaft, mit historischem Schwerpunkt (1993). Von dieser Entwicklung rührt das Interesse zum einen an theoretischen Fragen, zum anderen an Wort und Text als zentralen Bezugsgrößen meines Arbeitens.

\section{Frühneuzeitliche Sprachtheorie}

Das Zentrum meiner historischen Arbeit liegt in der Geschichte der Sprachtheorie, mit der Frühen Neuzeit als einem der Schwerpunkte. Diese Zeit ist vor allem deshalb interessant, weil mit der Aufwertung der Volkssprachen gegenüber dem Lateinischen, die sich von der Romania nach Norden ausbreitet, das Deutsche ab dem 16. Jahrhundert erstmals grammatikographisch und lexikographisch kodifiziert wird. Begleitet wird diese Entwicklung zum einen von der Reflexion über die Strukturen und kommunikativen Möglichkeiten der Volkssprache Deutsch, zum anderen über die 
Strukturen von Sprache im Allgemeinen. In beiden Fällen stehen die frühen Grammatiker und Sprachtheoretiker noch stark in der Tradition der Beschreibung des Lateinischen, das der lingua vulgaris Deutsch nicht nur als stilistisch überlegen gilt, sondern als eine Art lingua universalis auch das Modell für theoretische Beschreibungen von Sprache schlechthin abgibt.

Die Hinwendung zum Deutschen, der Drang zur Entwicklung einer leistungsstarken Hochsprache, erklärt sich aus zeitgeschichtlichen Zusammenhängen, aus den sich wandelnden politischen und ökonomischen Gegebenheiten (Territorialisierung, Kapitalisierung der Wirtschaft und Intensivierung des Handels etc.), den damit einhergehenden sozialen Verschiebungen (Herausbildung eines zunehmend einflussreichen Bürgertums), aus medialen Veränderungen (Entwicklung des Buchdrucks) und ihren Folgen, aus der Entstehung eines neuzeitlichen Wissenschaftsbegriffs mit der Notwendigkeit adäquater Fachsprachen, schließlich aus der Sicht des Deutschen als Medium des Ausdrucks politischer, gesellschaftlicher und kultureller Identität. In der Beschreibung der Strukturen des Deutschen beschränken sich erste Ansätze (z.B. Fabian Frangk 1531, Valentin Ickelsamer um 1534) mehr oder weniger auf orthographische Fragen, getragen von dem Bewusstsein, dass das deutschsprachige Gebiet aus wenigstens zwei geographischen Größräumen besteht, dem Nieder- und dem Oberdeutschen. Dabei zeigt nicht nur die Tatsache, dass viele der frühen Texte über das Deutsche in lateinischer Sprache verfasst sind, die enge Orientierung an den Lateingrammatiken von Donatus und Priscian, die über das gesamte Mittelalter hinweg den Sprachunterricht beherrschten. Auch kategorial ist diese Tradition zu erkennen, in der direkten Übertragung von strukturellen Merkmalen des Lateinischen auf das Deutsche, in der Grammatikographie z.B. des sechs Positionen umfassenden Kasussystems, in der Sprachpraxis aber auch von Formen, die zum festen Bestandteil des Systems des Deutschen wurden, ohne dass man sich dessen noch bewusst sein mag. (Wie groß auch immer der heutzutage gelegentlich beklagte Einfluss des Englischen auf die deutsche Sprache sein mag, er ist nicht einmal ansatzweise mit dem prägenden Einfluss des Lateinischen auf das Deutsche gleichzusetzen.)

Die in den Bereich der Stilistik und der Textgestaltung hineinreichenden Aspekte der frühneuzeitlichen Sprachnormierung sind der Tradition nicht der lateinischen Grammatik, sondern der antiken Rhetorik verpflichtet. Mit der Auffächerung des Spektrums an Textsorten, die die zunehmend divergenten Kommunikationsanlässe im öffentlichen Raum abdecken sollen, entstehen vor allem im 17. Jahrhundert Rhetoriken, Epistolographien, Titularien, Beschreibungen der Sekretariatkunst usw. in großer Zahl. Die dabei zugrunde gelegten Kriterien für gelungene Sprachverwendung - 
innertextuelle Kohärenz, situative Angemessenheit, Deutlichkeit, Orientierung am Sprachgebrauch vor allem herausgehobener Autoren, schließlich die Prinzipien des Aufbaus von Texten, auch unter dem Gesichtspunkt unterschiedlicher Textfunktionen wie Argumentieren, Beschreiben usw. - finden sich immer wieder im sprachbezogenen Schrifttum, bis zum Zurücktreten der Rhetorik aus dem Bildungskanon im 19. Jahrhundert. Die Untersuchung der Frage, ob auch unsere aktuellen Vorstellungen von gutem Deutsch nicht weit stärker von diesen traditionellen rhetorischen Kategorien geprägt sind, als wir denken, ist nach wie vor ein Desiderat der Forschung.

\section{Sprach- und Kulturpatriotismus}

Mit der Etablierung des Hochdeutschen (im geographischen wie im sozialen Sinne des Wortes) ergibt sich im 17. Jahrhundert erstmals die Möglichkeit, die gekonnte Beherrschung der Muttersprache zum Ausweis der Zugehörigkeit zu einer bestimmten gesellschaftlichen Gruppe zu erklären. Die Diskussion über diese Frage umfasst sehr unterschiedliche Positionen und wird ab dem frühen 17. Jahrhundert von kulturpatriotischen Anliegen überlagert. Dem Einfluss französischer Sprache und Lebensart an deutschen Höfen etwa wird von Mitgliedern der barocken Sprachgesellschaften ein Ideal der natürlichen, reinen, edlen deutschen Sprache entgegengehalten. Kennzeichnend für die zeitgenössische Argumentation ist die Enthistorisierung und Hypostasierung der deutschen Sprache, der, durch ihre Rückführung auf die babylonische Sprachverwirrung, biblisches Alter zuerkannt wird, zugleich aber auch eine unveränderliche Sprachnatur, ein genius linguae, der durch den Einfluss des Französischen und anderer romanischer Sprachen gefährdet sei. In einer für sprachpatriotische und -nationalistische Argumentationen charakteristischen Bewegung der Überblendung werden dabei Eigenschaften der Sprache auf solche der Sprecher übertragen: Wie das in germanischer Abfolge stehende Deutsch natürlich, rein und ontologisch-referentiell besonders zuverlässig (eigentlich) sei, gelten auch die in der Nachfolge der Germanen stehenden Sprecher des Deutschen als natürlich, aufrichtig und markant.

Jenseits des Germanenmythos und der Verquickung von Eigenschaften der Sprache mit solchen der Sprecher begegnet die Überzeugung, dass die kulturelle Identität von Sprachbenutzern durch fremden sprachlichen Einfluss gefährdet werden kann, in der Geschichte der Sprachreflexion immer wieder. Sie zeigt sich im antifranzösischen Sprachpurismus des 19. Jahrhun- 
derts ebenso wie in der gegenwärtigen Anglizismendebatte (dort natürlich ohne jeden Rückgriff auf den Germanenmythos und in moderater Form), auch in Sprachkulturen außerhalb der deutschen. Auch Gegenpositionen lassen sich immer finden, wie im 17. und im frühen 18. Jahrhundert durch die Fürsprecher einer Öffnung zu Frankreich, etwa Christian Thomasius und Gottfried Wilhelm Leibniz.

\section{Universalistische Ansätze}

Neben dieser auf das Deutsche als Einzelsprache gerichteten Linie der Sprachreflexion lässt sich in der Frühen Neuzeit eine universalistische nachweisen. Sie bildet das stärker analytisch und methodisch stringenter verfahrende Pendant zu den einzelsprachlichen Ansätzen, ohne gesellschaftliche Interessen und ideologische Überlagerung, auch ohne unmittelbaren Einfluss auf die Kodifizierungsbestrebungen. Die Rezeptionslinien zwischen den Ansätzen verlaufen quer durch Europa, die maßgeblichen Texte sind auf Latein verfasst. Dabei bildet das Lateinische nicht nur das Medium der Vermittlung universalistischer Theorien, sondern, zumindest zunächst, auch deren wissenschaftlichen Untersuchungsgegenstand. Bereits die mittelalterliche grammatica speculativa hat sich mit Fragen der Korrelation von sprachlichen Einheiten, solchen des Bewusstseins und Phänomenen der Realität befasst (vox/dictio/verbum-conceptus/notio-res). Diese Fragen werden nun aufgegriffen und führen zu interessanten Überlegungen über Möglichkeiten einer jenseits der oberflächenstrukturellen Unterschiede zwischen den Einzelsprachen bestehenden universalen sprachlichen Tiefenstruktur. Zwischen Oberflächen- und Tiefenstruktur werden Transformationen angesetzt, die z.B. synthetische Strukturen in analytischere überführen können (des Typs lego $=$ ego + legere $)$. In einer Verknüpfung solcher universalistischer Überlegungen mit einzelsprachlichen kommt es z.B. zwischen deutschen und französischen Grammatikern noch im 18. Jahrhundert zu Diskussionen darüber, ob das Deutsche oder aber das Französische in höherem Maße mit der natürlichen Ordnung der Dinge (ordre naturel/ordo naturalis) kongruent ist.

Die Sicht der Nationalsprachen als mehr oder weniger mangelhafter Varianten einer ihnen tiefenstrukturell zugrunde liegenden idealen Sprache führt ab dem 17. Jahrhundert zu Entwürfen künstlicher Sprachen. Sie sollen all die Mängel nicht aufweisen, die die natürlichen Sprachen kennzeichnen. In Deutschland hat unter anderem der Rationalist Leibniz, in England unter anderem der Empirist John Locke auf die vermeintlich unzureichende 
und das Denken irreführende sprachliche Kategorisierung der Welt in den Wortschätzen der Einzelsprachen hingewiesen: Die Bezeichnungen richteten sich nach der Gewohnheit der Sprecher, nicht aber nach der wahren Natur der Dinge. Künstliche Sprachen könnten die Relationen zwischen Sprache, Denken und Welt präzise abbilden, also etwa Erscheinungen wie Synonymie oder Polysemie vermeiden, könnten sich auch syntaktisch strukturgleich zur logischen Ordnung der Welt verhalten. In Äußerungen dieser Art zeigt sich ein analytischer Zugriff auf Sprache, wie er bis in die Ideal Language Philosophy des 20. Jahrhunderts besteht.

Auch wenn es auf den ersten Blick ungewöhnlich scheinen mag, muss in einer Skizze sprachuniversalistischer Ansätze der Frühen Neuzeit auch die Sprachmystik erwähnt werden. Sie teilt mit den bislang beschriebenen Ansätzen die Überzeugung einer jenseits der Einzelsprachen bestehenden ,Urform' von Sprache und betrachtet die Einzelsprachen als unzureichende Realisierungen dieser Urform. Bei der konkreten Bestimmung dieser sprachlichen Urform aber wie in ihrem gesamten argumentativen Duktus stellt die Sprachmystik das Gegenteil der oben beschriebenen universalistischen Ansätze dar. Im Werk Jacob Böhmes z.B. ist die Ursprache die Sprache des Paradieses, die durch Sündenfall und babylonische Sprachverwirrung der Menschheit verloren gegangen ist. Spuren (Signaturen) dieser ersten lingua Adamica aber sind in den Sprachen der Erde noch vorhanden und erschließen sich dem Blick des Visionärs. Wo im analytisch verfahrenden Sprachuniversalismus die Arbitrarität sprachlicher Zeichen als selbstverständlicher Ausgangspunkt allen Arbeitens begriffen wird und die ideale Sprache als eine von Menschen ins Werk gesetzte, perfekte Konstruktion gilt, geht die Sprachmystik von einer transzendenten Motiviertheit sprachlicher Zeichen aus, die auf die nie mehr erreichbare Sprache des Paradieses als ,eigentliche' und ideale Sprache der Menschheit verweisen.

\section{Texterschließung: Methodische Zugänge}

Sprachhistorische Forschung, die auf Zusammenhänge wie die geschilderten zugreifen will, muss sich über die Beschreibung des Sprachsystems hinaus- und in die Kulturgeschichte hineinbewegen. Das gilt nicht nur für Daten aus der Geschichte der Sprachtheorie, sondern auch für Befunde aus der Geschichte des Sprachsystems selbst wie auch seiner Verwendungsweisen. Eine in diesem Sinne kulturgeschichtlich arbeitende Sprachwissenschaft betrachtet sprachliche Phänomene vor dem Hintergrund gesellschaftlicher, politischer, ideengeschichtlicher, religionsgeschichtlicher, technisch-naturwissenschaftlicher, ästhetischer und alltagsweltlicher 
Zusammenhänge. Dabei sind Korrelationen zwischen kulturgeschichtlichen Entwicklungen und solchen im grammatischen Bereich wesentlich weniger unmittelbar gegeben als Korrelationen im Bereich von Wortschatz und Textgestaltung. Ziel der Analysen ist sowohl die Erschließung individueller Sprachverwendung (wobei „individuell“ auch die Verwendung durch mehr oder weniger umfangreiche gesellschaftliche Gruppen meinen kann) als auch der Beitrag dieser individuellen Verwendungsweisen zur Bildung fester Gebrauchsmuster innerhalb der Sprachgemeinschaft als Ganzer.

In der Frage der Erschließung der Textcorpora zeigen sich unterschiedliche Möglichkeiten. Will man sich nicht auf die methodisch ungeleitete Lektüre von Texten verlassen, die mehr oder weniger intuitiv auf inhaltliche Einzelaspekte zugreift, die dem Lesenden je nach Untersuchungsinteresse relevant erscheinen, dann bieten sich verschiedene sprachwissenschaftliche Analyseverfahren an, die in der neueren Forschung praktiziert werden. Sie greifen auf Kategorien der Grammatik, der Lexikologie, der Textlinguistik, der Rhetorik und Stilistik, in jüngster Zeit auch der Diskursanalyse zurück. Im Sinne einer Sprachgebrauchswissenschaft werden dabei die Corpustexte als in ihre konkreten Verwendungsbedingungen eingebunden begriffen. Es wird gefragt, wer diese Texte für welche Leser, in welcher Situation, mit welcher Intention, auf welche Weise verfasst hat (worin sich die Fragereihe von quis, quid, ubi, quibus auxiliis ... der zutiefst kommunikativ-pragmatisch orientierten antiken Rhetorik spiegelt).

Ein Analyseverfahren, das sich an lexikologischen Kategorien orientiert, ist die Begriffsgeschichte. In den Geisteswissenschaften umfassend bekannt geworden ist sie durch die "Geschichtliche[n] Grundbegriffe" von Otto Brunner, Werner Conze und Reinhart Koselleck (1972-1992). Die Feststellung der Herausgeber, dass ein zentraler Begriff sowohl „Indikator" als auch „Faktor" historischen Geschehens ist ${ }^{1}$, trifft das Anliegen genau: Begriffe - in der Sprachwissenschaft in der Regel verstanden als konzeptuelle Bündel von Bedeutungen semantisch benachbarter Ausdrücke - beschreiben einen Sachverhalt nicht einfach post festum, sondern bieten auch einen kategorisierenden Zugriff, eine jeweilige Perspektive auf diesen Sachverhalt, der bzw. die dessen künftige Wahrnehmung durch Sprachbenutzer in inhaltliche Bahnen lenkt. Dabei geht Begriffsgeschichte über Wortgeschichte insofern hinaus, als in der Analyse unterschiedliche Wörter herangezogen werden, die den jeweiligen Begriff konstituieren. Wer also z.B. den Begriff des Fremdworts in Texten des 17. und des

Reinhart Koselleck: Begriffsgeschichte und Sozialgeschichte. In: Ders. (Hrsg.): Vergangene Zukunft. Zur Semantik geschichtlicher Zeiten. Frankfurt 1979, S. 107-129; S. 120. 
18. Jahrhunderts untersucht, bezieht neben Fremdwort auch fremdes Wort, undeutsches Wort, Barbarismus usw. ein, wer Nation untersucht, wird auch Gemeinschaft, Volk, Stamm usw. berücksichtigen. ${ }^{2}$ In neueren sprachwissenschaftlichen Arbeiten wird zusätzlich das textuelle Umfeld eines Ausdrucks für die Analyse berücksichtigt, also dessen (partiellen) Synonyme, Antonyme, Hypo- und Hyperonyme, außerdem seine Einbindungen in Syntagmen unterschiedlicher Art (starke/gefährdetelfreie Nation; eine Nation schaffen/festigen/vereinen usw.). ${ }^{3}$

Die Untersuchung begriffsgeschichtlicher Zusammenhänge lässt sich über einzelne Textausdrücke hinaus in größere sprachliche Einheiten erweitern, zunächst in den Bereich der Präsuppositionen und Implikaturen. Auf diese Weise kann das im Text nur Mitbedeutete, aber nicht explizit Ausformulierte erfasst werden. Jenseits dieser Ebene wiederum lassen sich z.B. Topoi systematisch untersuchen. In Anlehnung an den rhetorischen Toposbegriff gelten Topoi dabei als Bündelungen kollektiven Wissens zu einem thematischen Sachverhalt, deren Beschreibung einen Zugriff auf die argumentative Struktur von Texten und das in ihnen sedimentierte Wissen erlaubt. ${ }^{4}$

Die größte Einheit der Untersuchung stellt der Diskurs dar, verstanden als Summe von Texten zu einem Thema, die das Wissen und die Einstellungen der gesellschaftlichen Gruppen, die die Texte verfassen, zu diesem Thema spiegeln und zugleich handlungsleitend für den zukünftigen Umgang damit sind. Diskursanalysen können unter sich die genannten Methoden vereinen, müssen dabei aber auch die intertextuellen Bezüge berücksichtigen, also die Verbindungen zwischen den einzelnen Texten und Textgruppen, die Übernahme oder Ablehnung von semantischen Komponenten einzelner Begriffe. ${ }^{5}$

Wird nicht gezielt nach individuellen Textausdrücken bzw. Begriffen gesucht, bieten sich Verfahren der Schlagwort- oder der Metaphernanalyse

2 Zur Illustration des Verfahrens s. z.B.: Anja Lobenstein-Reichmann: Houston Stewart Chamberlain - Zur textlichen Konstruktion einer Weltanschauung. Eine sprach-, diskurs- und ideologiegeschichtliche Analyse. Berlin/New York 2009.

3 Z.B. Katja Faulstich: Konzepte des Hochdeutschen. Der Sprachnormierungsdiskurs im 18. Jahrhundert. Berlin/New York 2009; Szilvia Odenwald-Varga: „Volk“ bei Otto von Bismarck. Eine historisch-semantische Analyse anhand von Bedeutungen, Konzepten und Topoi. Berlin/New York 2009; Marcus Müller: Geschichte - Kunst - Nation. Die sprachliche Konstituierung einer ,deutschen' Kunstgeschichte aus diskursanalytischer Sicht. Berlin/New York 2007.

4 Z.B. Martin Wengeler: Topos und Diskurs. Begründung einer argumentationsanalytischen Methode und ihre Anwendung auf den Migrationsdiskurs (1960-1985). Tübingen 2003.

5 Z.B. Ingo Warnke (Hrsg.): Diskurslinguistik nach Foucault. Theorie und Gegenstände. Berlin/New York 2007. 
an. ${ }^{6}$ Den meisten der beschriebenen Ansätzen ist gemein, dass sie die Bedeutungsbildung in Texten als ein flächiges Phänomen begreifen, das durch unterschiedliche sprachliche Formen - auch solche grammatischer Natur realisiert wird. In jüngster Zeit wird versucht, Ergebnisse der kognitiven Linguistik für die Textanalyse fruchtbar zu machen. Dabei stehen Wissensagglomerationen im Zentrum der Analyse (Frames), die als im Wesentlichen sprachlich konstituiert gelten. ${ }^{7}$

All diese Analyseverfahren sind sog. qualitative Verfahren, bei denen der Analysierende selbst die Corpustexte auf bestimmte Aspekte hin untersucht. In beschränktem Umfang lassen sie sich ergänzen durch quantitative Verfahren. In ihnen werden digital aufbereitete Texte mittels eigens dafür entwickelter Software gezielt auf einzelne Ausdrücke abgefragt, in einer Weise, die auch die Prädikationen zu diesen Ausdrücken, also ihre semantische Qualifizierung erkennen lässt ${ }^{8}$. Bei großen Datenmengen erlauben solche Verfahren einen interessanten ersten Zugriff auf ein Textcorpus, der jedoch durch individuelle Analysen ergänzt werden muss.

Die genannten Methoden sind hinsichtlich der zeitlichen Spezifik der Corpustexte neutral. Sie lassen sich zur Analyse älterer Texte ebenso einsetzen wie zu Analysen aktueller Sprachverwendung, sei es der Diskurs über die Computerisierung unseres Alltags ${ }^{9}$, sei es die Beschreibung künstlerischer Zusammenhänge im Rahmen einer Ausstellung wie der Documenta. ${ }^{10}$

6 Z.B. Anja Stukenbrock: Sprachnationalismus. Sprachreflexion als Medium kollektiver Identitätsstiftung in Deutschland (1617-1945). Berlin/New York 2005; Claudia Fraas: SchlüsselKonzepte als Zugang zum kollektiven Gedächtnis - Ein diskurs- und frameanalytisch basierter Ansatz. In: Deutsche Sprache 3/2005, S. 242-257.

7 Zur Begrifflichkeit vgl. z.B. Ekkehard Felder: Semantische Kämpfe in Wissensdomänen. Eine Einführung in Benennungs-, Bedeutungs- und Sachverhaltsfixierungs-Konkurrenzen. In: Ders. (Hrsg.): Semantische Kämpfe. Macht und Sprache in den Wissenschaften. Berlin/New York 2006, S. 13-46; zur Frame-Theorie: Alexander Ziem: Frames und sprachliches Wissen. Kognitive Aspekte der semantischen Kompetenz. Berlin/New York 2008.

8 Z.B. Noah Bubenhofer: Sprachgebrauchsmuster. Korpuslinguistik als Methode der Diskursund Kulturanalyse. Berlin/New York 2009.

9 Albert Busch: Diskurslexikologie und Sprachgeschichte der Computertechnologie. Tübingen 2004.

10 Andreas Gardt: Kunst und Sprache. Beobachtungen anlässlich der Documenta 12. In: Literatur Kunst - Medien. Festschrift für Peter Seibert zum 60. Geburtstag. Hrsg. von Achim Barsch, Helmut Scheuer und Georg-Michael Schulz. München 2008, S. 201-224. 